\title{
The Proposal of Directed Graph Evaluation Method for Engineering Risks
}

\author{
LIU Qiujie
}

Sichuan University of Arts and Science, Dazhou 635006, Sichuan

\begin{abstract}
Keywords: Engineering Risks; Risk Events (Factors); Directed Graph Evaluation Method; Maximum Probability Chain; Maximum Loss Chain
\end{abstract}

\begin{abstract}
In this paper, directed graph evaluation method for engineering risks was proposed on the basis of identifying and estimating engineering risks: the overall causation between the risks of engineering projects was visually expressed using directed graphs. The concept of the maximum probability chain and the concept of the loss chain as well as their algorithms were proposed in directed graphs. By solving the maximum probability chain and the maximum loss chain of the risk factors (bottom risk reasons), the key risk events and the key risk factors in engineering projects were determined, thus providing a powerful basis for the construction units to make accurate decisions and the risk managers to take reasonable preventive measures.
\end{abstract}

\section{Introduction}

Engineering risks refer to those risks, which possibly appear in the stages such as decision-making, design, construction, and completion acceptance in engineering projects, go against the goals of all units participating in the engineering, and also may give rise to the uncertainties such as personal injuries, property loss, or other economic loss. The bases of the evaluation on engineering risks are risk identification and risk estimation. In the risk identification, the risks in engineering projects were first concluded and classified (according to the internal and external factors of engineering projects, technical and non-technical factors, engineering project targets, or all subjects), for the purpose of identifying risk events, showing the causes of the risk events respectively, and then further investigating the causes until specific measures were taken. That is to say, all different risk events (risk factors) were independent of each other and had no connection with each other, as shown in Fig.1 (the classification according to all subjects of engineering projects). 


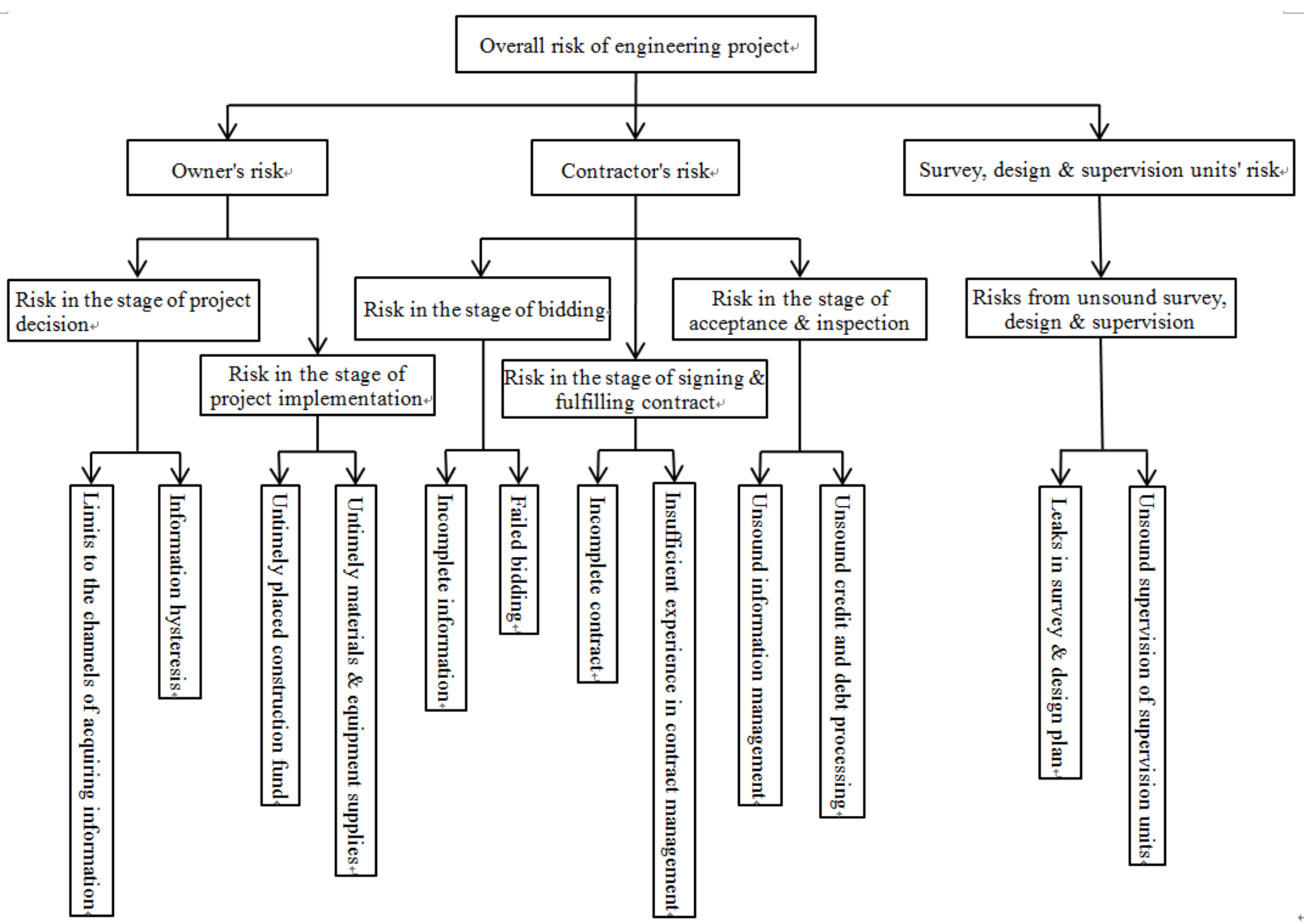

Fig.1 the causal relationship between independent risk factors in each category

However, in the risk management of some specific engineering projects, the risk events (risk factors) in each category were interrelated and restricted, thus forming a complex relational network of risks, as shown in Fig.2. 


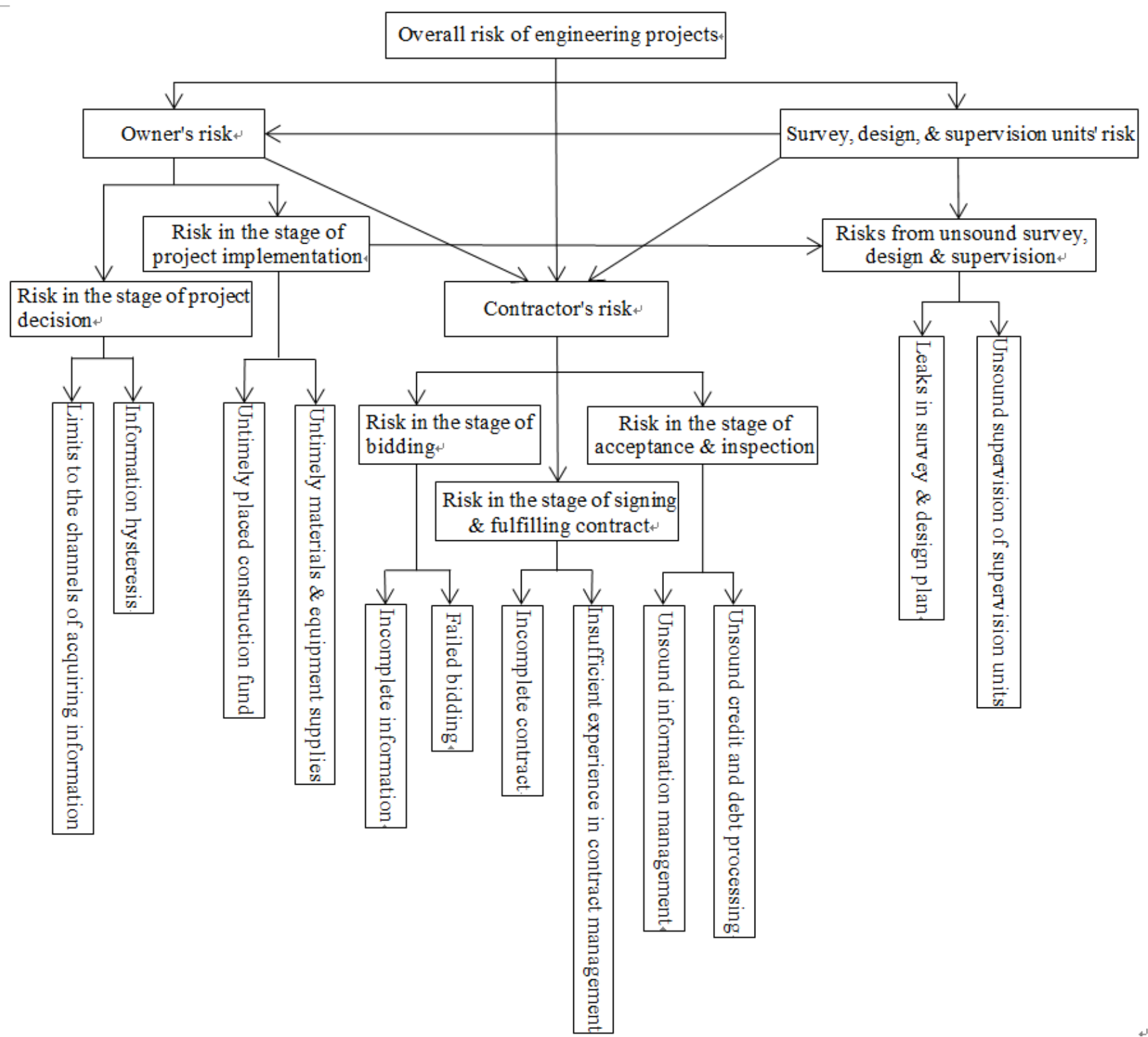

Fig. 2 the causal relationship between connected risk factors in each category

Then, the evaluation on the risks of engineering project in the risky relationship similarly shown in Fig.2 was the key point to be discussed in this paper.

\section{The Proposal of Directed Graph Evaluation Method}

Engineering risk evaluation refers to comprehensively analyzing the engineering risks on the basis of risk identification and estimation and sorting the risk levels according to the influence degree of risks on engineering targets. Engineering risk evaluation aims to uncover the key risks of engineering projects according to the risk evaluation, identify the overall evaluation level of engineering, and provide a basis for making risk response plans.

Directed Graph. In real life, the graphs composed of the ligatures between points were used for reflecting a specific relationship between some objects, in which the arrow-contained ligature between two points was called as arc.

If graph D was composed of points and arc, it was called as a directed graph [1], expressed as $D=(V, A)$, in which $V$,A were the point set and arc set of $D$ respectively. The one direction was the arc from $v_{i}$ to $v_{j}$, expressed as $\left(v_{i}, v_{j}\right)$.

The overall risk relationship of the engineering project shown in Fig.2 was expressed with a directed graph shown in Fig.3: points $v_{1}, v_{2}, \ldots . ., v_{22}$ represented the studied risk events or factors; the 
arc between points meant there was a causal relationship between two risk events (risk factors). For example, arc $\left(v_{i}, v_{j}\right)$ meant $v_{j}$ was a cause of $v_{i}$. If each arc in Fig.3 was weighted, it was a weighted directed graph.

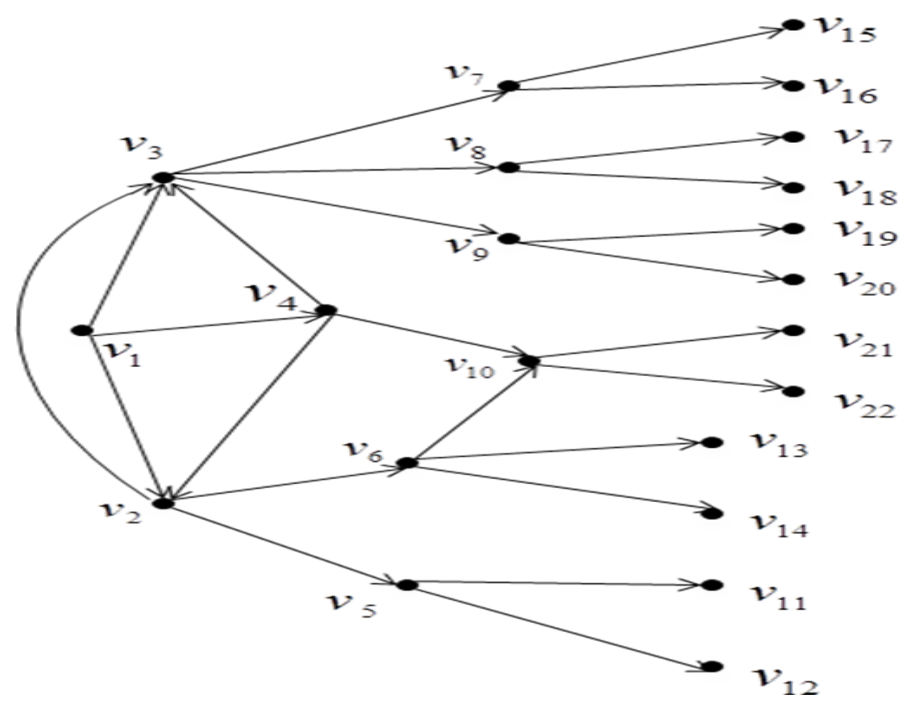

Fig.3 directed graph for engineering risks

$\mathrm{v}_{1}$ : overall risk of engineering project; $\mathrm{v}_{2}$ : owner's risk; $\mathrm{v}_{3}$ : contractor's risk; $\mathrm{v}_{4}$ : Survey, design, \& supervision units' risk; $\mathrm{v}_{5}$ : risk in the stage of project decision; $\mathrm{v}_{6}$ : Risk in the stage of project implementation; $\mathrm{v}_{7}$ : Risk in the stage of bidding; $\mathrm{v}_{8}$ : Risk in the stage of signing \& fulfilling contract; $\mathrm{V}_{9}$ : Risk in the stage of acceptance \& inspection; $\mathrm{v}_{10}$ : risks from unsound survey, design \& supervision; $\mathrm{v}_{11}$ : Limits to the channels of acquiring information; $\mathrm{v}_{12}$ : information hysteresis; $\mathrm{v}_{13}$ : untimely placed construction fund; $\mathrm{v}_{14}$ : Untimely materials \& equipment supplies; $\mathrm{v}_{15}$ : Incomplete information; $\mathrm{v}_{16}$ : failed bidding; $\mathrm{v}_{17}$ : Incomplete contract; $\mathrm{v}_{18}$ : Insufficient experience in contract management; $\mathrm{v}_{19}$ : unsound information management; $\mathrm{v}_{20}$ : unsound credit and debt processing; $\mathrm{v}_{21}$ : Leaks in survey \& design plan; $\mathrm{v}_{22}$ : Unsound supervision of supervision units

Maximum Probability Chain and Its Algorithm. A weighted directed graph was given, meaning a directed graph $D(V, A)$ was given; to each $\operatorname{arc} a=\left(v_{i}, v_{j}\right)$, a related weight $w(a)=p_{t}$ was available, in which $p_{t}$ was the probability of risk factor $v_{j}$ to occur for the risk events of an upper layer; two vertices $v_{s}, v_{t}$ of $D$ were also given. It was assumed that $P$ was a road in $D$ from $v_{s}$ to $v_{t}$; the weight of the road $P$ was the product of the weights of all the arcs of $P$, expressed as $\mathrm{s}_{w(P)}$. The problem of the maximum probability chain was to solve a road with the maximum weight from the roads $v_{s} \sim v_{t}$, meaning a road $P_{0}$ from $v_{s}$ to $v_{t}$ was solved.

$$
w\left(P_{0}\right)=\max _{P} w(P)
$$

Where, the maximum value of the road $P$ from $v_{s}$ to $v_{t}$ was taken, meaning $P_{0}$ was the maximum probability chain from $v_{s}$ to $v_{t}$. The weight of the road $P$ was the probability of risk factor $v_{t}$ to occur for risk event $v_{s}$, expressed as $p\left(v_{s}, v_{t}\right)$.

The basic idea of the algorithm of the maximum probability chain started from $v_{s}$ (top risk event) and then gradually explored the maximum probability chain outward. In the execution process, a number corresponding to each point was recorded, expressing the weight (i.e. the label of $P$ ) of the maximum probability chain from $v_{s}$ to this point, or the lower bound (i.e. the label of $T$ ) of the weight of the maximum probability chain rom $v_{s}$ to this point; in each step, the label of $T$ was modified, and the point with the label of $T$ was changed to the point with the label of $P$, thus there was one more vertex with the label of $P$ in $D$. Then, the maximum probability chain could be solved from $v_{s}$ to all points by forwarding at most $p-1$ steps. 
In the specific steps, $P, T$ were used for expressing some point's label of $P$ and label of $T$ respectively; $s_{i}$ was the set of the points with the label of $P$ at step $i$. In order to solve the maximum probability chain from $v_{s}$ to all points, a value of $\lambda$ was given to each point $v$; if $\lambda(v)=m$ at the termination of the algorithm, the former point of $v$ was $v_{m}$ in the maximum probability chain from $v_{s}$ to $v$; if $\lambda(v)=M$, the road from $v_{s}$ to $v$ was not contained in $D ; \lambda(v)=0$ meant $v=v_{s}$.

The specific steps for solving the maximum probability chain: a weighted directed graph $D=(V, A)$ was given.

( $i=0$ ) at the beginning; order $S_{0}=\left\{v_{s}\right\}, P\left(v_{s}\right)=1, \lambda\left(v_{s}\right)=0$; to each $v \neq v_{s}, \operatorname{order}_{T}(v)=0, \lambda(v)=M$, and $k=s$.

(1)If $S_{i}=V$, the algorithm was terminated; then, $v \in S_{i}, p\left(v_{s}, v\right)=P(v)$; otherwise, the algorithm enters (2);

(2)Every point $v_{j}$ making $\left(v_{\mathrm{k}}, v_{j}\right) \in A \& v_{j} \notin S_{i}$ was examined;

If $T\left(v_{j}\right)<P\left(v_{k}\right) \bullet w_{k j}, T\left(v_{j}\right)$ was modified as $P\left(v_{k}\right) \bullet w_{k j}$, and $\lambda\left(v_{j}\right)$ was revised as $k$; otherwise, the algorithm enters (3);

(3) Order $T\left(v_{j_{i}}\right)=\max _{v_{j} \notin S_{i}}\left\{T\left(v_{j}\right)\right\}$

If $T\left(v_{j_{i}}\right)>0$, the label of $T$ of $v_{j_{i}}$ was changed to the label of $P P\left(v_{j_{i}}\right)=T\left(v_{j_{i}}\right)$; order $S_{i_{1+1}}=S_{i} \cup\left\{v_{j_{i}}\right\}, k=j_{i}$, in which $i$ was changed to $i+1$, and then algorithm enters (1); otherwise, the algorithm was terminated. Then, $v \in S_{i}, p\left(v_{s}, v\right)=P(v)$ and $v \notin S_{i}, p\left(v_{s}, v\right)=T(v)$ were established.

Maximum Loss Chain and Its Algorithm. A weighted directed graph was given, meaning a directed graph $D(V, A)$ was given; to each $\operatorname{arc} a=\left(v_{i}, v_{j}\right)$, a related weight $w(a)=w_{t}$ was available, in which $w_{t}$ was the loss caused by risk factor $v_{j}$ to engineering project; two vertexes $v_{s}, v_{t}$ in $D$ were given.

It was assumed that $P$ was a road in $D$ from $v_{s}$ to $v_{t}$; the weight of the road $P$ was the sum of all the arcs of $P$, expressed with ${ }_{w(P)}$. The problem of the maximum loss chain was to solve a road with the maximum weight from all roads $v_{s} \sim v_{t}$, meaning a road $P_{0}$ from $v_{s}$ to $v_{t}$ was solved to make the following equation established:

$$
w\left(P_{0}\right)=\max _{P} w(P)
$$

Where, the maximum value of the road $P$ from $v_{s}$ to $v_{t}$ was taken, meaning $P_{0}$ was the maximum loss chain from $v_{s}$ to $v_{t}$.

The basic idea of the algorithm of the maximum loss chain started from $v_{s}$ (top risk event) and gradually explored the maximum loss chain outward. In the execution process, a number corresponding to each point was recorded, and called as the label of this point, which was used for expressing the weight (i.e. the label of $P$ ) of the maximum loss chain from $v_{s}$ to this point, or the lower bound (i.e. the label of $T$ ) of the weight of the maximum loss chain from $v_{s}$ to this point; in each step, the label of $T$ was modified, and every point with the label of $T$ was changed to the point with the label of $P$, thus there was one more vertex with the label of $P$ in $D$. Then, the maximum loss chain could be solved from $v_{s}$ to all points by forwarding at most ${ }_{p-1}$ steps.

In the specific steps, $P, T$ were used for expressing some point's label of $P$ and label of $T$ respectively; $S_{i}$ was the set of the points with the label of $P$ at step $i$. In order to solve the maximum loss chain from $v_{s}$ to all points, a $\lambda$ value was given to every point $v$; if $\lambda(v)=m$ at the termination of the algorithm, the former point of $v$ was $v_{m}$ in the maximum loss chain from $v_{s}$ to $v$; if $\lambda(v)=M$, the road from $v_{s}$ to $v$ was not contained in $D ; \lambda(v)=0$ meant $v=v_{s}$. 
The specific steps for solving the maximum loss chain: a weighted directed graph $D=(V, A)$ was given.

( $i=0$ ) at the beginning; order $S_{0}=\left\{v_{s}\right\}, P\left(v_{s}\right)=0, \lambda\left(v_{s}\right)=0$; to each $v \neq v_{s}$, order $T(v)=0, \lambda(v)=M$, and $k=s$.

(1) If $S_{i}=V$, the algorithm was terminated; otherwise, the algorithm entered (2);

(2)The point $v_{j}$ making $\left(v_{\mathrm{k}}, v_{j}\right) \in A \& v_{j} \notin S_{i}$ was examined;

If $T\left(v_{j}\right)<P\left(v_{k}\right)+w_{k j}, T\left(v_{j}\right)$ was modified as $P\left(v_{k}\right)+w_{k j} ; \lambda\left(v_{j}\right)$ was revised as $k$; the algorithm entered (3).

(3) Order $T\left(v_{j_{i}}\right)=\max _{v_{*} \in S_{i}}\left\{T\left(v_{j}\right)\right\}$

If $T\left(v_{j_{i}}\right)>0$, the labél of $\mathrm{T}$ of $v_{j_{i}}$ was changed to the label of $\mathrm{P} P\left(v_{j_{i}}\right)=T\left(v_{j_{i}}\right)$, and order $S_{i+1}=S_{i} \cup\left\{v_{j_{i}}\right\}, k=j_{i}$, in which $i$ was converted to $i+1$, and then the algorithm entered (1); otherwise, the algorithm was terminated.

Directed Graph Evaluation Method. On the basis of risk identification and estimation of engineering risks and combining with the theories of maximum probability chain and maximum loss chain, the basic steps of the directed graph evaluation method proposed in this paper for engineering risks are as follows:

(1) The causations between various identified risks were expressed with a directed graph; two weighted directed graphs were formed using the probability of occurrence of risk events (risk factors) and the loss weight caused by engineering projects, namely, the probability weight directed graph and the loss weight directed graph.

(2) In the probability weight directed graph and the loss weight directed graph, the maximum probability chain and the maximum loss chain of risk factors (bottom risk causes) were solved respectively.

(3) The sorting of the comprehensive risk factors in the occurrence probability of the overall engineering project risk and the loss caused by the whole engineering project were analyzed to find the risk factors (bottom risk causes) requiring key focus and the common risk events on the maximum probability chain and the maximum loss chain.

(4) Measures and plans were made in a targeted way according to key risk events and factors for effectively avoiding risks and reducing the influence of risks on engineering project.

\section{Case Analysis}

In the engineering project with a risk relationship shown in Fig.2, it was assumed that the probability of all risk events (risk factors) to occur for the risk events of an upper layer and the loss caused by all risk events (risk factors) to the whole construction project were as shown in Fig.4.

$\square$ expressed the top risk event (the overall risk of engineering project) and intermediate risk events; $\bigcirc$ expressed risk factors (bottom risk causes); the numbers in the rectangle and the circular expressed the loss (unit: yuan) caused by each risk event (risk factor) to the whole engineering project; the number nearby each arc expressed the probability of each risk event (risk factor) to occur for risk events of an upper layer. 


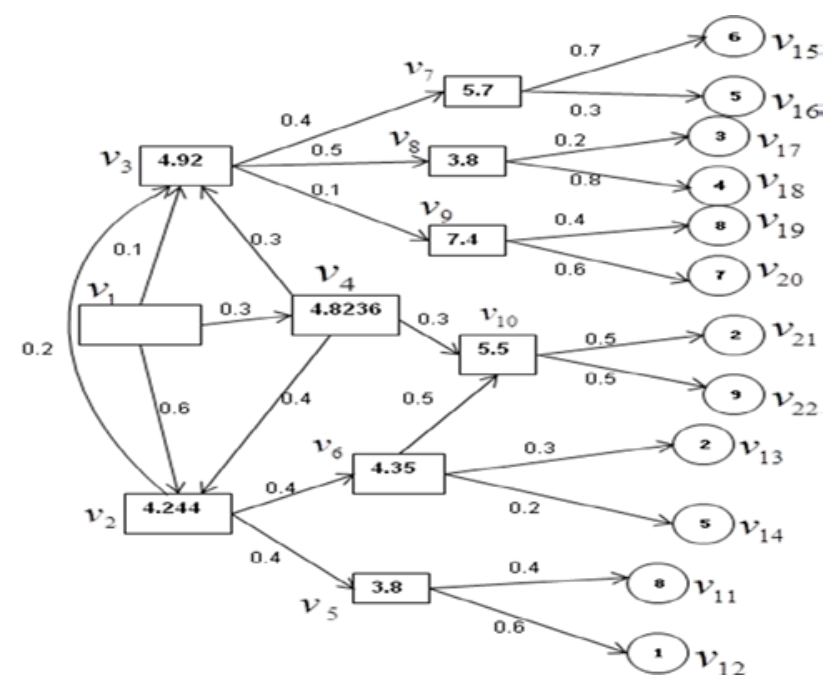

Fig.4 direct graph for the possiblity of engineering risks to occur and the related losses

Solving the Maximum Probability Chain. The engineering project with a risk relationship shown in Fig.4 was expressed with a weighted directed graph (Fig.5) for the distribution of the probabilities; the number nearby each arc expressed the possibility of each risk event (risk factor) to occur for the risk events of an upper layer. The maximum probability chain was solved from $v_{1}$ to $_{t} v_{t}(t=11,12,13,14, \ldots \ldots ., 22)$.

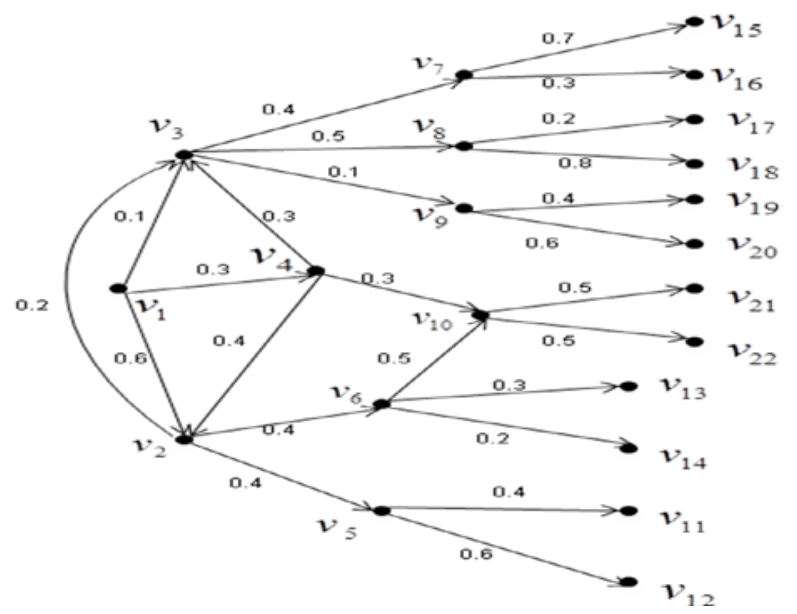

Fig.5 weighted directed graph for the possibility of engineering risks to occur

The maximum possibility chain from $v_{1}$ to $v_{11}$, solved with the algorithm in section 1.2 , was $\left(v_{1}, v_{2}, v_{5}, v_{11}\right)$, and the total weight was 0.096 ; the maximum possibility chain from $v_{1}$ to $v_{12}$ was $\left(v_{1}, v_{2}, v_{5}, v_{12}\right)$, and the total weight was 0.144 ; the maximum possibility chain from $v_{1}$ to $v_{13}$ was $\left(v_{1}, v_{2}, v_{6}, v_{13}\right)$, and the total weight was 0.072 ; the maximum possibility chain from $v_{1}$ to $v_{14}$ was $\left(v_{1}, v_{2}, v_{6}, v_{14}\right)$, and the total weight was 0.048 ; the maximum possibility chain from $v_{1}$ to $v_{15}$ was $\left(v_{1}, v_{2}, v_{3}, v_{7}, v_{15}\right)$, and the total weight was 0.0336 ; the maximum possibility chain from $v_{1}$ to $v_{16}$ was $\left(v_{1}, v_{2}, v_{3}, v_{7}, v_{16}\right)$, and the total weight was 0.0144 ; the maximum possibility chain from $v_{1}$ to $v_{17}$ was $\left(v_{1}, v_{2}, v_{3}, v_{8}, v_{17}\right)$, and the total weight was 0.012 ; the maximum possibility chain from $v_{1}$ to $v_{18}$ was $\left(v_{1}, v_{2}, v_{3}, v_{8}, v_{18}\right)$, and the total weight was 0.048 ; the maximum possibility chain from $v_{1}$ to $v_{19}$ was $\left(v_{1}, v_{2}, v_{3}, v_{9}, v_{19}\right)$,and the total weight was 0.0048 ; the maximum possibility chain from $v_{1}$ to $v_{20}$ was $\left(v_{1}, v_{2}, v_{3}, v_{9}, v_{20}\right)$, and the total weight was 0.0072 ; the maximum possibility chain from $v_{1}$ to $v_{21}$ was $\left(v_{1}, v_{2}, v_{6}, v_{10}, v_{21}\right)$, and the total weight was 0.06 ; the maximum possibility chain from $v_{1}$ to $v_{22}$ was $\left(v_{1}, v_{2}, v_{6}, v_{10}, v_{22}\right)$, and the total weight was 0.06 . 
Therefore, the possibilities of the risk factors $v_{11}, v_{12}, v_{13}, v_{14}, \ldots \ldots, v_{22}$ to occur from the overall risk of engineering project $v_{1}$ were sorted, and the result is as shown below:

$$
p\left(v_{1}, v_{12}\right)>p\left(v_{1}, v_{11}\right)>p\left(v_{1}, v_{13}\right)>p\left(v_{1}, v_{21}\right)=p\left(v_{1}, v_{22}\right)>p\left(v_{1}, v_{14}\right)=p\left(v_{1}, v_{18}\right)>p\left(v_{1}, v_{15}\right)>p\left(v_{1}, v_{16}\right)>p\left(v_{1}, v_{17}\right)>p\left(v_{1}, v_{20}\right)>p\left(v_{1}, v_{19}\right)
$$

Solving the Maximum Loss Chain. The engineering project with a risk relationship shown in Fig.4 was expressed with a weighted directed graph (Fig.6) for the distribution of the losses; the number nearby each arc expressed the loss caused by each risk event (risk factor) to the whole engineering project. The maximum loss chain was solved from $v_{1}$ to $_{t}(t=11,12,13,14, \ldots . . ., 22)$.

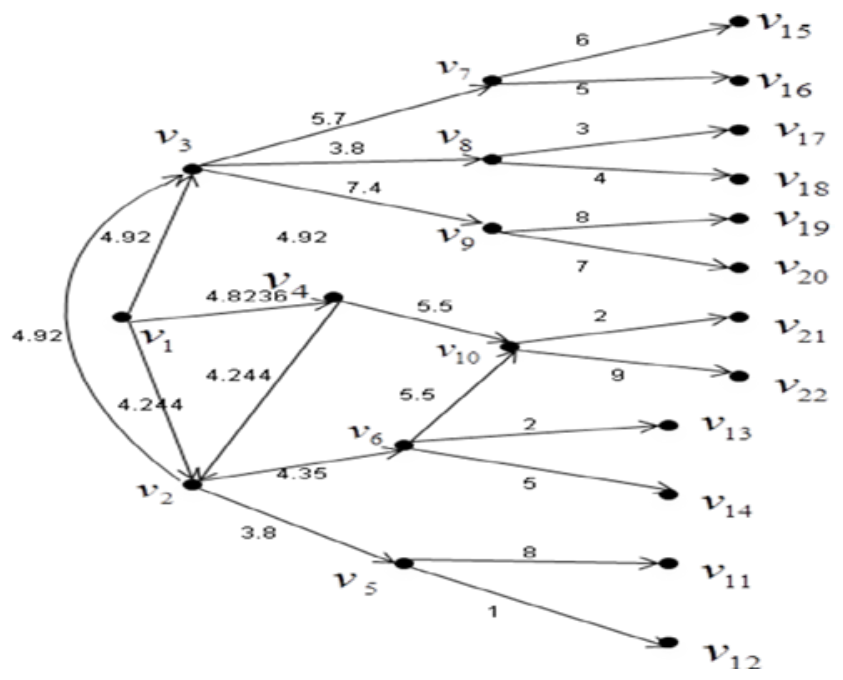

Fig.6 weighted directed graph for the losses caused by engineering risks

The maximum loss chain from $v_{1}$ to $v_{11}$, solved with the algorithm in section 1.3, was $\left(v_{1}, v_{4}, v_{2}, v_{5}, v_{11}\right)$; the maximum loss chain from $v_{1}$ to $v_{12}$ was $\left(v_{1}, v_{4}, v_{2}, v_{5}, v_{12}\right)$; the maximum loss chain from $v_{1}$ to $v_{13}$ was $\left(v_{1}, v_{4}, v_{2}, v_{6}, v_{13}\right)$; the maximum loss chain from $v_{1}$ to $v_{14}$ was $\left(v_{1}, v_{4}, v_{2}, v_{6}, v_{14}\right)$; the maximum loss chain from $v_{1}$ to $v_{15}$ was $\left(v_{1}, v_{4}, v_{2}, v_{3}, v_{7}, v_{15}\right)$; the maximum loss chain from $v_{1}$ to $v_{16}$ was $\left(v_{1}, v_{4}, v_{2}, v_{3}, v_{7}, v_{16}\right)$; the maximum loss chain from $v_{1}$ to $v_{17}$ was $\left(v_{1}, v_{4}, v_{2}, v_{3}, v_{8}, v_{17}\right)$; the maximum loss chain from $v_{1}$ to $v_{18}$ was $\left(v_{1}, v_{4}, v_{2}, v_{3}, v_{8}, v_{18}\right)$; the maximum loss chain from $v_{1}$ to $v_{19}$ was $\left(v_{1}, v_{4}, v_{2}, v_{3}, v_{9}, v_{19}\right)$; the maximum loss chain from $v_{1}$ to $v_{20}$ was $\left(v_{1}, v_{4}, v_{2}, v_{3}, v_{9}, v_{20}\right)$; the maximum loss chain from $v_{1}$ to $v_{21}$ was $\left(v_{1}, v_{4}, v_{2}, v_{6}, v_{10}, v_{21}\right)$; the maximum loss chain from $v_{1}$ to $v_{22}$ was $\left(v_{1}, v_{4}, v_{2}, v_{6}, v_{10}, v_{22}\right)$.

Comprehensive Evaluation Analysis. The sorting of the possibilities of comprehensive risk factors $v_{11}, v_{12}, v_{13}, v_{14}, \ldots . ., v_{22}$ to occur for the overall risk $v_{1}$ of engineering project and the losses caused by risk factors $v_{11}, v_{12}, v_{13}, v_{14}, \ldots . ., v_{22}$ to the whole engineering project were analyzed for identifying the key risk factors $v_{11}, v_{14}, v_{15}, v_{18}, v_{22}$ and the common risk events (risk factors) between the maximum possibility chain and the maximum loss chain; the existing risk causal relationship between these factors is as shown below:

$$
v_{2} \rightarrow v_{5} \rightarrow v_{11}, v_{2} \rightarrow v_{6} \rightarrow v_{14}, v_{2} \rightarrow v_{3} \rightarrow v_{7} \rightarrow v_{15}, v_{2} \rightarrow v_{3} \rightarrow v_{8} \rightarrow v_{18}, v_{2} \rightarrow v_{6} \rightarrow v_{10} \rightarrow v_{22}
$$

As shown in Fig.7, 


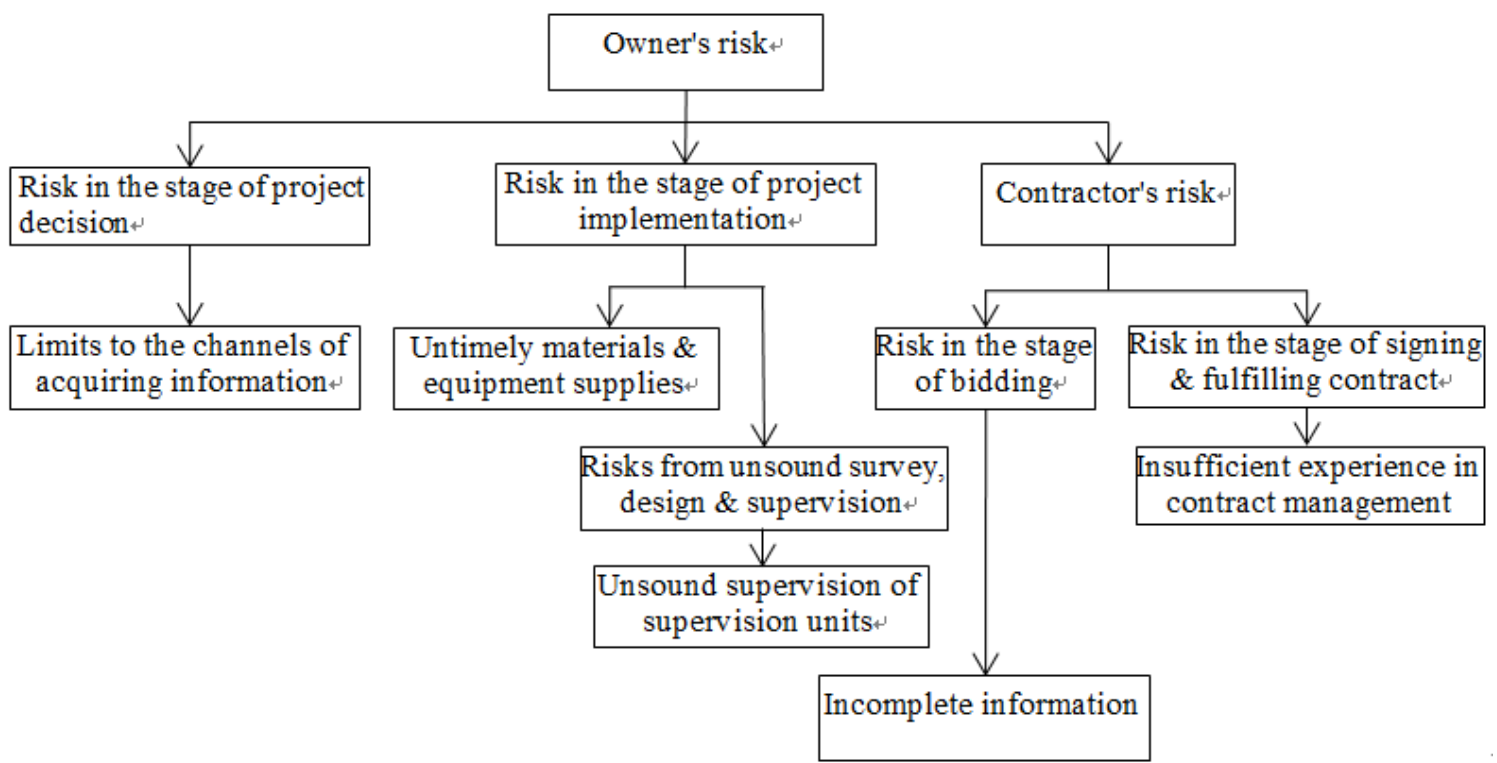

Fig.7 the causal relationship between key risk events (risk factors)

The final analysis showed that "owner's risk" was the risk event exerting the largest effect on the whole engineering project; construction units (owners) needed to actively expand the channels of acquiring the information in the stage of project decision-making, and ensure the timely supplies of materials and equipment, strengthen the supervision of the supervision units and the bidding information completeness and contract management systematism of contractors, so as to reduce the occurrence probability of the overall risk of engineering project and also the losses to the maximum degree.

\section{Conclusion}

In this paper, the directed graph evaluation method proposed for engineering risks was to identify the key risk events and factors of the whole engineering project on the basis of analyzing the causes of key risk events (risk factors) layer by layer using flow charts, visually expressing the relationship using directed graph (simply the relational network), and solving the maximum possibility chain and the maximum loss chain, so as to take related measures in a targeted way and reduce the overall risk level of engineering project.

The advantages of directed graph evaluation method were concluded from the following several aspects.

First, the expression for the causal relationship between the risks of engineering projects using directed graph was simple, vivid, clear, and easy to operate.

Second, the probabilities of comprehensive risk events (risk factors) to occur in the whole engineering project and the caused losses were used in the risk evaluation, and thus the limitations of a single evaluation way were avoided, and also the overall risk of engineering project was fully, reasonably evaluated.

Third, the maximum probability chain and the maximum loss chain were proposed. The maximum probability of risk factors (bottom risk causes) to occur in the overall risk of engineering project was effectively solved using the maximum probability chain, thus laying a foundation for sorting the risks.

Fourth, by integrating the maximum probability chain and maximum loss chain of risk factors (bottom risk causes) together, the key risk events and factors of engineering project could be definitely identified, thus providing a powerful basis for risk managers to carry out risk analysis and formulate measures. 


\section{References}

[1] Chief Editor of the Teaching Materials Compilation Group for Operational Research. Operational Research [M]. Tsinghua University Press (Ed.3).

[2] JIAO Yonglan. Management Operational Research [M]. China Railway Publishing House, 2005.

[3] REN Xu. Project Risk Management [M]. Tsinghua University Press/Beijing Jiaotong University Press, 2010.

[4] DENG Xuecai, YI Wanlong. Engineering Project Risk Management. VIP Information.

[5] WU Yun'na. The Whole Process Project Management Planning of Engineering Construction [J]. Chinese Consulting Engineers, 2007, (10).

[6] PAN Yuxin, CUI Linlin. Discussion on Construction Project Risk Management [J]. Project Management, 2009.

[7] ZHOU Jianguo. Engineering Project Management [M]. Beijing: China Power Press, 2006. 